\title{
Detection of Klebsiella pneumoniae Carbapenemase (KPC) and Metallo-Beta Lactamase (MBL) Producing Gram Negative Bacteria Isolated from Different Clinical Samples in A Transplant Center, Kathmandu, Nepal
}

\author{
Krishna Dhungana ${ }^{1}$, Bal Krishna Awal ${ }^{2}$, Binod Dhungel ${ }^{1}$, Supriya Sharma ${ }^{1}$, Megha Raj Banjara ${ }^{1}$ and Komal Raj \\ Rijal $^{1 *}$ \\ ${ }^{1}$ Central Department of Microbiology, Tribhuvan University, Kathmandu, Nepal \\ ${ }^{2}$ Human Organ Transplantation Centre, Bhaktapur, Nepal \\ *Corresponding Author: Komal Raj Rijal, Central Department of Microbiology, Tribhuvan University, Kirtipur, Kathmandu, Nepal.
}

Received: October 21, 2019; Published: November 11, 2019

DOI: $10.31080 /$ ASMI.2019.02.0432

\begin{abstract}
Multidrug-resistant Gram-negative bacteria are associated with higher mortality and morbidity among solid organ transplant recipients. Carbapenems, the broad spectrum $\beta$ lactam agents are frequently used as a drug of last resort for the treatment of the infections caused by MDR Gram negative bacteria. They are the cause of production of $\beta$ lactamases (Klebsiella pneumoniae Carbapenemase-KPC and Metallo $\beta$ lactamases-MBL) among Gram negatives. The objective of this study was to explore the present scenario of $\beta$-lactamases mainly KPC along with MBL productions in Gram negative clinical samples. This hospital based cross sectional study was conducted from January 2016 to June 2016 at Human Organ Transplant Center, Kathmandu, Nepal.
\end{abstract}

The standard microbiological methods were used for the isolation and identification of all the isolates and screening the meropenem resistant isolates by Kirby Bauer Disc Diffusion method. The confirmatory detection of KPC and MBL was done by the $\beta$-lactamase inhibition disc method. The inhibiting compound Phenyl Boronic Acid (PBA) and Ethylenediamine tetra-acetic Acid (EDTA) were used. Out of 1500 different clinical samples processed, only 10\% (15/1500) Gram-negative organisms were isolated and identified. MDR was detected in 77.3\% isolates. Majority of the Meropenem resistant (50\%) isolates being E. coli (69.3\%) followed by $K$ pneumoniae (18.6\%). Carbapenemase MBL was prevalent among 29\% of the isolates while KPC was reported among $12.6 \%$ isolates.

The study demonstrated the increase in trend of carbapenemase production is creating turmoil in treatment options in health centers. This study put forward a prospective need for the development of such drugs or other mechanisms which could better work to overcome the resistance due to carbapenemase production.

Keywords: Gram Negative Bacteria (GNB), $\beta$-lactamases, Klebsiella pneumoniae Carbapenemase (KPC), Metallo $\beta$-Lactamases (MBL), Inhibition Disc Method

\section{Abbreviations}

AMR: Antimicrobial Resistance; MDR: Multi-Drug Resistance/Resistant; ESBL: Extended Spectrum Beta-Lactamase; ATCC: American Type Culture Collection; CLSI: Clinical Laboratory Standard Institute; CFU: Colony Forming Unit; UTI: Urinary Tract Infection $\mathrm{ABc}$ : AmpC Beta- Lactamases; KPC: Klebsiella pneumoniae Carbapenemases; BA: Blood Agar; BHI: Brain Heart Infusion; MBL: Metallo Beta lactamases; CDC: Center for Disease Control and prevention; CSF: Cerebrospinal Fluid; MA: Mac Conkey Agar; MHA: Muller Hinton Agar; SPSS: Statistical Package for Social Sciences; WHO: World Health Organization; PCR: Polymerase Chain Reaction; NDM: New Delhi metallo Beta lactamase

\section{Introduction}

Although organ transplants patients are particularly susceptible to a number of infections, the most frequent group isolated so far is Enterobacteriaceae--Escherichia and Klebsiella being the two representative ones, which can lead to increased cost and longer duration of hospitalization [1,2].

Beta- lactams comprise the largest group of antibacterial including penicillin's, cephalosporins, carbapenems, and monobactams commonly targeted against infectious Gram-negative bacteria (GNB). Carbapenems (meropenem, imipenem, biapenem, doripenem, razupenem and ertapenem) often used to treat bacterial in- 
fections caused by Gram-positives, Gram-negatives, and anaerobes are parenteral synthetic $\beta$-lactams derived from thienamycin, an antibiotic produced by Streptomyces cattleya [3-5]. They are often used as empirical therapy to treat bacterial infections caused by Gram- positives, Gram-negatives and anaerobic bacteria and are known to be stable against almost all of the beta-lactamases, including Amp C and extended-spectrum beta-lactamases (ESBL), which are involved in enzymatic resistance in bacteria [5-7]. Betalactamases are the enzymes produced by bacteria that hydrolyze the beta- lactam ring, thereby inactivating the drug [8].

Carbapenems are member of molecular class A, class B and Class D $\beta$-lactamases. Class A and D enzymes have serine basedhydrolytic mechanism, while class B enzymes are metallo- $\beta$ lactamases that contain zinc in the active site which can hydrolyse almost all $\beta$-lactams and most are resilient against inhibition by all commercially viable $\beta$-lactamase inhibitors [9] and can spread among bacterial strains and species [7]. A unique MBL, the New Delhi metallo- $\beta$-Lactamase (NDM-1), has emerged from this class and is proving to be a problematic mechanism because of its ability to inactivate all $\beta$-lactam antibiotics except for aztreonam [9-11].

$\beta$-lactamase that hydrolyze carbapenems have become prominent these days notably Klebsiella pneumonia carbapenems (KPC) and MBL such as the New Delhi metallo $\beta$-lactamase (NDM) [1214]. NDM-1 is an enzyme which can inactivate carbapenems and other $\beta$-lactams. The gene encoding for the enzyme is mainly found among GNB belonging to the family Enterobacteriaceae. These enzymes are usually encoded on plasmids that harbor multiple resistance determinants and are transmitted easily from one Enterobacteriaceae to another because of the ease of transfer of genetic material by plasmids [15]. NDM- 1 is a novel $\beta$-Lactamases and is the latest threat to medical fraternity [16].

Although K. pneumoniae remains the most prevalent bacterial species carrying KPCs, the enzyme has been identified in several other Gram-negative bacilli [17]. MBL-mediated resistance has appeared in several pathogenic strains in the last 20 years and is being rapidly spread by horizontal transfer, involving both plasmid and integron-borne genetic elements. Presently, there are no clinically useful inhibitors for MBLs which is serious clinical threat to the $\beta$-lactam antibiotic therapy [18].

The risk factors attributed for carbapenems production can be following: previous exposure to broad-spectrum antibiotics, especially the third-generation cephalosporins and carbapenems, previous use of fluoroquinolone prophylaxis, serious illness (e.g. end-stage disease, sepsis, pneumonia), nosocomial infection, prolonged hospital stay and/or repeated hospitalizations, urinary catheters, older age, intensive care unit stay [19-21].
Carbapenemase gene detection by molecular methods is the gold standard but is available in only a few reference laboratories, and phenotypic tests have therefore been developed. The modified Hodge test (MHT) is the only Clinical and Laboratory Standards Institute (CLSI) recommended carbapenemase-screening method (CLSI 2014).

In developing countries like Nepal, very few researches on the importance and antibiogram of carbapenems are currently available, however, certain researches have assessed the antibiogram of carbapenems in special reference to ESBLs reported that $100 \%$ of E. coli, M. morgani and E. aerogens were susceptible to both imipenem and meropenem whereas K. pneumoniae revealed $6.86 \%$ and $10.78 \%$ resistance to imipenem and meropenem respectively [22]. Many carbapenem resistant isolates were found in the sputum and endotracheal secretions in which $100 \%$ Klebsiella species were MDR and among them $4.2 \%$ were MBL producers [23]. Quite high rates of MBL production among Gram negative bacteria [24]. The presence of NDM-1 MBL producing Providencia rettgeri has also been reported in specimens obtained at study in Kathmandu [25]. Also, $15 \%$ of $E$. coli was detected to be MBL producer in another study [26]. In another study, $18.98 \%$ of the E. coli and $21.08 \%$ of the K. pneumoniae were found to be carbapenemase producers [27]. In recent study conducted at Kathmandu Model hospital the prevalence of carbapenem resistance among the bacteria belonging to Enterobacteriaceae ranged from $4.49 \%$ to $20 \%$ [28].

Infections with multidrug-resistant GNB are associated with higher mortality and morbidity among solid organ transplant recipients [29]. Production of carbapenemases (KPC, MBL) has been the global cause of Carbapenem resistance among Gram negative bacteria, which is a great therapeutic challenge. The increasing and rapid spread of carbapenem resistance has not been yet accessed fully in context of Nepal, so this study was undertaken to ascertain the present scenario of $\beta$ lactamases mainly KPC along with MBL productions in Gram-negative isolates obtained through clinical samples in a transplant center.

\section{Methods}

This hospital based cross-sectional prospective study was carried out from January 2016 to July 2016 at Human Organ Transplant Center, Bhaktapur, Nepal.

Patients who had undergone dialysis treatment, those with Immune suppression clinical intervention and those suspected of infections were included as study subjects. Patients under the treatment of antibiotic course were excluded in this study. After routine culture testing, only Gram-negative isolates were considered for further analysis. 
A total of 1500 different including urine, sputum, pus and tips were collected and processed by standard microbiological methods [30]. Out of 1500 samples collected estimated 150(10\%) culture positive samples for Gram negative bacteria was included in the study. Mid-stream urine was collected in a sterile, dry, widenecked leak- proof container. Cleansing of genatalia was done to reduce contamination. In case of pus and wound swab, pus was aspirated in syringe by trained medical personnel. If pus was not discharging, cotton swab was gently rolled over the surface of the wound approximately five times, focusing on area where there was evidence of pus or inflamed tissue. Two swabs were taken from each patient, one for culture and another for direct gram staining. In case of Sputum, deep cough free from saliva and nasal discharge was collected in a sterile, leak-proof, disposable container. CVP and Double J Stent tubes in renal pelvis and urinary bladder tips were removed and clipped about 2 inches of the distal end directly into a sterile container [30,31].

The collected samples were labeled properly and immediately delivered to laboratory for further processing. When immediate delivery was not possible, the specimen was refrigerated at $4-60$ C [32].

\section{Laboratory examinations of samples}

Microscopic examination

Smears of specimens like pus were prepared; gram stained and observed through microscope for the presence of bacteria and pus cells.

\section{Culture}

For processing of each sample, microbiological protocols were followed [30,31]. Using a sterile calibrated loop, a loopful of samples was inoculated on MacConkey agar (MA) and Blood agar (BA) then incubated aerobically at $37^{\circ} \mathrm{C}$ overnight. But in case of tips, Catheter tips were first incubated on BHI broth at $37 \mathrm{C}$ for 24 hours and sub-cultured on MA and BA plates and incubated at $37 \mathrm{C}$ overnight.

After adequate incubation of culture plates, colony count was done, significant result was considered for plates showing more than or equal to 105 colony forming units (CFU)/ml of urine.

\section{Identification of isolates}

Presumptive identification isolates were done on the basis of colony colour and Gram's staining morphology. Then, obtained pure cultures of isolates were assessed for biochemical tests for confirmation $[30,31]$.

\section{Antibiotic susceptibility testing (AST)}

All identified isolates of $E$. coli and Klebsiella species were treated for susceptibility testing against Amikacin $(30 \mu \mathrm{g})$, Amoxicillin
$(10 \mu \mathrm{g})$, Azithromycin $(15 \mu \mathrm{g})$, Cefixime $(5 \mu \mathrm{g})$, Ceftriaxone $(30 \mu \mathrm{g})$, Ciprofloxacin $(5 \mu \mathrm{g})$, Cotrimoxazole $(25 \mu \mathrm{g})$, Cefoperazone $(75 \mu \mathrm{g})$, Cefoperazone sulbactum $(75 / 30 \mu \mathrm{g})$, Gentamicin $(10 \mu \mathrm{g})$, Meropenem $(10 \mu \mathrm{g})$, Polymixin B $(10 \mu \mathrm{g})$ Nalidixic acid $(30 \mu \mathrm{g})$, Nitrofurantoin $(300 \mu \mathrm{g})$, Piperacillin-Tazobactam $(100 / 10 \mu \mathrm{g})$, and Tobramycin $(10 \mu \mathrm{g})$ (Hi-Media India Pvt. Ltd) following Kirby-Bauer method on Mueller-Hinton Agar (Hi-media India Pvt. Ltd.). Results were interpreted using CLSI guidelines (CLSI, 2014). Those isolates which were non susceptible (either a resistant or intermediate) to three or more antibiotics classes were considered as multi-drug resistant [33].

\section{Screening for carbapenemase producers}

If the zone size was less than $15 \mathrm{~mm}$ for Meropenem (CLSI 2014) then they were subjected for KPC and MBL phenotypic tests.

\section{Phenotypic confirmatory test for carbapenemase producers}

Inhibitor-based method was followed for the confirmation of KPC and MBL detection. Phenylboronic acid (PBA) and Ethylene Diamine Tetra Acetic Acid (EDTA) were inhibitors used for the confirmatory detection of KPC and MBL respectively [34].

\section{Phenotypic confirmatory test for KPC producers}

Boronic acid compounds are serine-type $\beta$-lactamase inhibitors that were employed originally for the detection of KPC producing Gram negative isolates. In that respect, combined disc tests using carbapenems with and without phenylboronic acid (PBA) have been proposed as the most accurate phenotypic tests for detecting KPC production [34,35]. The screened carbapenemase producers were tested for confirmatory KPC production by using this combined disc and Meropenem alone and the increase in diameter of zone of inhibition $\geq 5 \mathrm{~mm}$ in PBA supplemented meropenem against meropenem disc was confirmed as KPC production and result was interpreted [36].

\section{Phenotypic confirmatory test for MBL producers}

For this test, the $\beta$-lactam disk (meropenem) was potentiated with an inhibitor EDTA, and the diameter of its inhibition zone was then compared with that of the $\beta$-lactam disk alone, hence the term "combined disk test" (CDT).

This test detected the presence of specific enzyme containing zinc at its active site i.e. metallo betalactamase [37]. Two meropenem disks $(10 \mu \mathrm{g})$ were taken, one containing $10 \mu \mathrm{l}$ of $0.1 \mathrm{M}$ EDTA. Disks were placed $25 \mathrm{~mm}$ apart on the test organism inoculated MHA plates and incubated. An increase in zone diameter of $>5 \mathrm{~mm}$ around the meropenem-EDTA disk compared to that of meropenem alone was considered positive for MBL production [14].

\section{Quality control}

Each batch of media and reagents were subjected to sterility and performance testing. During AST, quality control was done us- 
ing the control strains of E. coli ATCC 25922. Strict adherence to aseptic conditions and laboratory safety was adopted during each steps of examination.

Data were entered and analyzed by using Statistical Package for Social Science (SPSS) version 21 software package. Relation of variables was analyzed using ratio and percentage.

\section{Result}

A total of 1500 different clinical samples including urine (1168), sputum (160), pus (30) and tips (142) were processed, out of which $150(10 \%)$ samples showed gram negative bacterial growth. Out of 150 culture positive samples processed, 104 (69.3\%) E. coli was most frequent isolated followed by K. pneumoniae 28 (18.7\%), P. mirabilis 4 (2.7\%) sample P. vulgaris 2 (1.3\%), P. aeruginosa 6 (4\%) C. freundii 2 (1.3\%) and Providencia species 4 (2.7\%).

\section{Distribution of MDR in Gram negative organisms}

In this study the prevalence of multi drug resistant (MDR) among Gram negative isolates was found to be $77.3 \%$. Total of 18 antibiotics of different classes were tested for which all isolates of $P$. vulgaris, C. freundii and Providencia species were found to be MDR while only $79.8 \%$ and $60.7 \%$ of $E$. coli and $K$. pneumoniae isolates were found to be MDR (Figure 1).

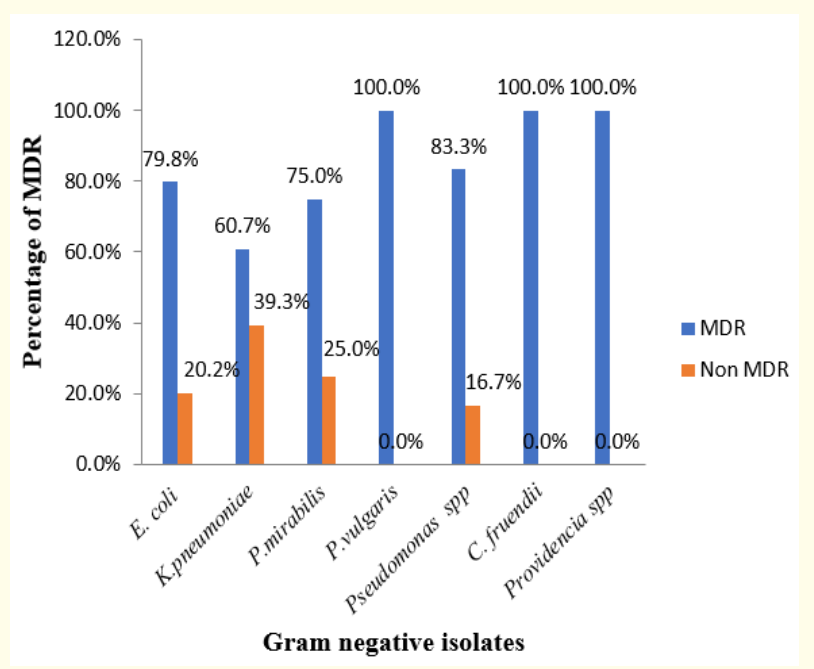

Figure 1: Distribution of MDR among Gram negative organisms.

\section{Distribution of KPC among Gram negative organisms}

Altogether $19(12.6 \%)$ isolates were found to be KPC producers including E. coli, K. pneumoniae, P. aeruginosa and C. freundii. Among all KPC producers $78.9 \%$ were E. coli, $10.5 \%$ K. pneumoni$a e$ and rest other isolates P. vulgaris, P. mirabilis and Providencia species were detected as non KPC organisms (Figure 2).

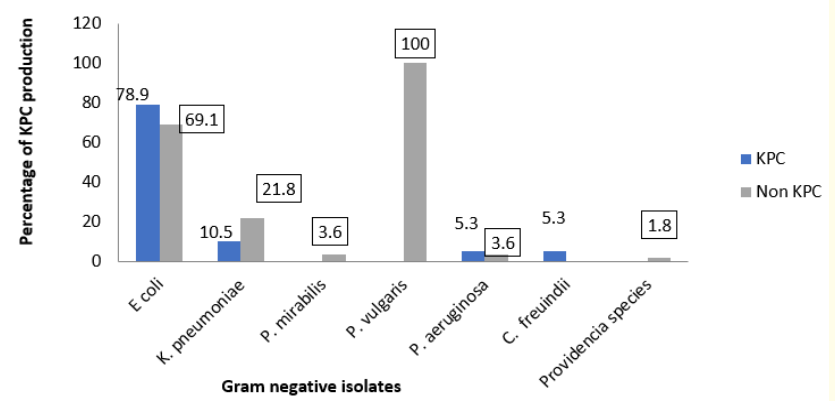

Figure 2: Distribution of KPC in Gram negative organisms.

\section{Distribution of KPC among various samples}

KPC producing isolates were obtained from urine (78.9\%), tips (15.8\%) and pus (5.3\%). The most frequent KPC producers obtained from urine sample was E. coli (57.8\%) followed by $10.5 \%$ $K$. pneumoniae. From pus and tips sample only KPC producing $E$. coli (5.3\% and $15.8 \%$ ) were obtained. KPC producing P. aeruginosa (5.3\%) and C. freundii (5.3\%) were isolated only from urine sample (Figure 3).

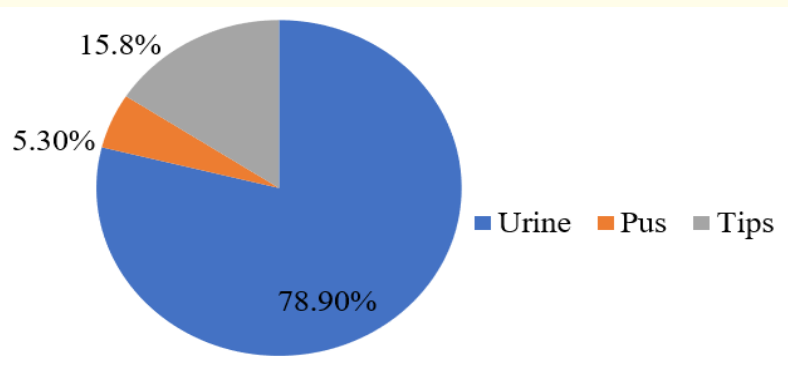

Figure 3: Distribution of KPC among various samples.

\section{Distribution of KPC in various age groups and gender}

KPC producing isolates were found higher in females (63.2\%) than in males (37.8\%). It was detected that KPC producing isolates were found to be highest in the age group 40 - 45 years which accounts for $31.6 \%$ while least was observed in age group $70-80$ years $(5.3 \%)$ (Figure 4$)$.

Distribution of MBL production in gram negative organisms

Altogether 44 (29.3\%) isolates were found to be MBL producers. Except $P$. vulgaris all the isolated Gram-negative organisms were MBL producers. The highest number of MBL producers isolated were E. coli (63.6\%) and prevalence of MBL in E. coli was found to be $26.9 \%$. Similarly, the prevalence of MBL production in K. pneumoniae was found to be $39.3 \%$. The prevalence of MBL in $C$. 


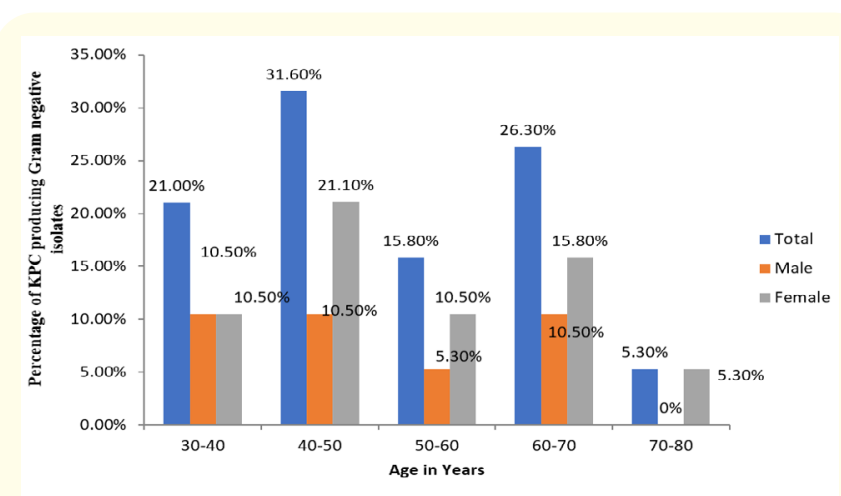

Figure 4: Distribution of KPC in various age groups and gender.

freundii was found to be the highest of $50 \%$. The overall prevalence of MBL among Gram negative from different clinical specimens like urine, pus, sputum and tips obtained in the transplant center was 29.3\%. Statistically there was no association between Gram negative isolates and MBL production ( $p=0.360$ ) (Table 1$)$.
Sex-wise distribution of MBL in gram negative organism

MBL producing isolates were obtained from all samples. MBL producing isolates were high in male $(54.5 \%)$ than in female (45.5\%). The majority of MBL producers in male and female were isolated from the urine sample. In female $45 \%$ of MBL producers were isolated from urine while in male $54.3 \%$ were isolated from urine. No isolates were found to be producing MBL in pus of male and sputum of female (Table 2).

Age-wise distribution of MBL production by gram negative organisms

MBL producing isolates were obtained from all samples. Highest MBL production was detected in urine sample (79.5\%) followed by tips sample (13.6\%). MBL production was detected high in Gram negative bacteria isolated from age group 40 - 50 years $(31.8 \%)$ with in the urine sample (37.1\%). 100\% MBL production was demonstrated in the pus sample collected from age group 50 - 60 years. The minimum percentage of MBL production was observed in age

\begin{tabular}{|c|c|c|c|c|c|c|c|c|}
\hline \multirow{3}{*}{ Organisms } & \multicolumn{2}{|c|}{ MBL Screen } & \multicolumn{4}{|c|}{ MBL Profile } & \multirow{3}{*}{ Total isolate } & \multirow{3}{*}{ p-Value (Isolates/MBL production) } \\
\hline & \multirow[b]{2}{*}{ No } & \multirow[b]{2}{*}{$\%$} & \multicolumn{2}{|c|}{ Producer } & \multicolumn{2}{|c|}{ Non-Producer } & & \\
\hline & & & No & $\%$ & No & $\%$ & & \\
\hline E. coli & 56 & 53.8 & 28 & 26.9 & 25 & 24.1 & 104 & \\
\hline K. pneumoniae & 14 & 50 & 11 & 39.2 & 3 & 10.7 & 28 & \\
\hline P. mirabilis & 2 & 50 & 1 & 25 & 1 & 25 & 4 & \\
\hline P.vulgaris & 2 & 100 & 0 & 0 & 2 & 100 & 2 & \\
\hline $\begin{array}{l}\text { Pseudomonas } \\
\text { spp }\end{array}$ & 3 & 50 & 2 & 33.3 & 1 & 16.7 & 6 & $0.360^{*}$ \\
\hline C.freuindii & 1 & 50 & 1 & 50 & 0 & 0 & 2 & \\
\hline $\begin{array}{l}\text { Providencia } \\
\text { spp }\end{array}$ & 1 & 25 & 1 & 25 & 0 & 0 & 4 & \\
\hline Total & 76 & 50.7 & 44 & 29.3 & 32 & 21.3 & 150 & \\
\hline
\end{tabular}

Table 1: Distribution of MBL production in Gram negative organisms. *Chi-square test.

\begin{tabular}{|l|l|l|l|l|l|}
\hline \multirow{3}{*}{ Sample } & \multicolumn{4}{c|}{ MBL Profile } & \multirow{2}{*}{ Total } \\
\cline { 2 - 5 } & \multicolumn{2}{|c|}{ Female } & \multicolumn{2}{c|}{ Male } & \\
\cline { 2 - 5 } & No & \multicolumn{1}{c|}{$\%$} & No & \% & \\
\hline Urine & 16 & 45 & 19 & 54.3 & 35 \\
\hline Sputum & 0 & 0 & 2 & 100 & 2 \\
\hline Pus & 1 & 50 & 0 & 0 & 2 \\
\hline Tips & 3 & 50 & 3 & 50 & 6 \\
\hline Total & 20 & 45.5 & 24 & 54.5 & 44 \\
\hline
\end{tabular}

Table 2: Sex-wise distribution of MBL.

\begin{tabular}{|c|c|c|c|c|c|c|}
\hline \multirow{2}{*}{$\begin{array}{c}\text { Age } \\
\text { (Years) }\end{array}$} & \multicolumn{5}{|c|}{ MBL Profile } & \multirow{2}{*}{$\begin{array}{c}\text { p- } \\
\text { Value }\end{array}$} \\
\hline & $\begin{array}{l}\text { MBL } \\
\text { (\%) }\end{array}$ & $\begin{array}{c}\text { Urine } \\
(\%)\end{array}$ & $\begin{array}{c}\text { Sputum } \\
\text { (\%) }\end{array}$ & $\begin{array}{l}\text { Pus } \\
(\%)\end{array}$ & $\begin{array}{l}\text { Tips } \\
\text { (\%) }\end{array}$ & \\
\hline $0-10$ & $2(4.5)$ & $2(5.7)$ & 0 & 0 & 0 & \multirow[t]{9}{*}{$0.31^{*}$} \\
\hline $10-20$ & $1(2.2)$ & 0 & 0 & 0 & $1(16.6)$ & \\
\hline $20-30$ & $6(13.6)$ & $4(11.4)$ & $1(50)$ & 0 & $1(16.6)$ & \\
\hline $30-40$ & $4(9.1)$ & $3(8.6)$ & 0 & 0 & $1(16.6)$ & \\
\hline $40-50$ & $14(31.8)$ & $13(37.1)$ & 0 & 0 & $1(16.6)$ & \\
\hline $50-60$ & $9(20.5)$ & $7(20)$ & 0 & $1(100)$ & 1(16.6) & \\
\hline $60-70$ & $6(13.6)$ & $4(11.4)$ & $1(50)$ & 0 & $1(16.6)$ & \\
\hline $70-80$ & $2(4.5)$ & $2(5.7)$ & 0 & 0 & 0 & \\
\hline Total & $44(100)$ & $35(79.5)$ & $2(4.5)$ & $1(2.2)$ & $6(13.6)$ & \\
\hline
\end{tabular}

Table 3: Age wise Distribution of MBL production. 
group 10 - 20 years which is only $2.2 \%$. The prevalence of MBL production among age group 9 - 79 years from different samples like urine, pus, sputum and tips are $79.5 \%, 4.5 \%$ ), $2.2 \%$ and $13.6 \%$ respectively. The statistical analysis of the data demonstrated that there is no significant association between age group and MBL production $(\mathrm{p}=0.310)$ (Table 3$)$.

\section{Discussion}

This study was performed at Human Organ Transplant Center, Bhaktapur to detect the prevalence of Beta-lactamase producing Gram-negative isolates from all clinical samples obtained in the center. Beta-lactamases are now disseminating on mobile genetic elements among clinically important Gram-negative pathogens which are associated with infections resulting higher rates of mortality, morbidity, and health care costs. Carbapenems are used as the drugs of last resort for treatment of MDR Gram-negative bacterial infection.

In this study, altogether 7 different bacterial isolates were obtained where most common pathogens were E. coli and K. pneumoniae. Of the total 1500 different clinical samples processed, 150 (10\%) samples showed low positive microbial growth. Low culture positivity has been reported from different specimens in the past studies which is analogous to this study also [38-40]. The common factors behind the low growth rate observed might be due to the use of antibiotics prior to sample collection, success of infection control system strategy aimed to prevent diseases and control of bacterial infection within the hospitals [41]. It also might be due to anaerobic bacteria since only aerobic incubation technique is used [42]. E. coli (69.3\%) and K. pneumoniae (18.7\%) were most frequently isolated Gram-negative bacteria. Comparably similar isolation rate for the organism from different samples were observed in the past study [43] but lower rate was reported in another study [40]. E. coli and K. pneumoniae are clinically important members of the family Enterobacteriaceae commonly causing infections of different sites [44, 45].

In this study the prevalence of MDR among Gram negative isolates were found to be $77.3 \%$. All isolates of P. vulgaris, C. freundii and Providencia spp. were found to be MDR while only $79.8 \%$ and $60.7 \%$ of $E$. coli and K. pneumoniae isolates were found to be MDR. Higher rate of MDR in this study is comparable to some past studies $[26,46]$ whereas lower rate was observed in other studies [24,47]. However, the difference in MDR rates between the present and the other studies could not be compared due to varying definitions of MDR used during categorizing isolates into MDR. Various mechanism of resistance like low outer membrane permeability, production of an AmpC $\beta$-lactamase, metallo $\beta$-lactamases, mutations to topo-isomerases II and IV that confer fluoro-quinolone resistance and the presence of numerous genes coding for different multidrug resistance efflux pumps as well as a high number of acquired resistance genes coding for amino-glycoside modifying enzymes and $\beta$-lactamases has contributed in evolution of multidrug resistance organisms $[48,49]$. MDR and presence of several virulence factors in the strains of many pathogens responsible to different diseases has increased a threat to the successful disease management.

Since there is no standard guideline for detection of MBL production, different studies have reported to have used different methods. The inhibition disc technique was used for the detection of carbapenemase (KPC and MBL) production in gram negative organisms. In our study the beta - lactamase inhibitors EDTA and PBA were used for the detection of KPC and MBL respectively which is similar to the so called combined dics test (CDT) using meropenem in combination with inhibitors. In the evaluation of two selected MBL phenotypic assays (CDT and DDST), CDT was found to be more sensitive (16.98\%), than DDST (13.2\%).

Double disk synergistic test was used in this study for the confirmation of KPC and MBL production in the Gram-negative isolates. In most of the studies, these inhibition tests are used for MBL and KPC detection along with MHT [16,37] while in recent years, E-tests using Imipenem/ Imipenem-EDTA are also being used for their detection $[50,51]$.

Among these methods for the detection of MBL and KPC producers, (inhibition test) was found to be more effective for their phenotypic detection than Modified Hodge test. During this study all meropenem resistant isolates were detected by inhibition test and the sensitivity was calculated to be $84 \%$ (among 75 meropenem resistant isolates suspected of producing carbapenemase only 44 were detected to be MBL producers and only 19 were detected to be KPC producers by inhibition technique). Similar result was found in an Indian study where 118 (86.5\%) isolates were detected by inhibition technique [16, 37].

In this study the average carbapenemase production was found to be $12.6 \% \mathrm{KPC}$ and $26.3 \% \mathrm{MBL}$ among the Gram-negative isolates. The prevalence of MBL production in E. coli was reported as $29.33 \%$ (28/104) and in K. pneumoniae 39.2\% (11/28). Similarly, KPC production in E coli was reported as $14.4 \%$ (15/104) and $7.1 \%(2 / 28)$ in K. pneumoniae. The findindg is in harmony with some past studies in Nepal $[24,28]$ and abroad [10, 52,53]. Quite high rates of AmpC $\beta$-lactamase (37.2\%) and MBL (36.7\%) production (among gram negative bacteria) in comparison to our study were also reported [24]. As revealed from study attempted to detect the frequency of carbapenemase producing K. pneumoniae in Dhaka city of Bangladesh in 2011, out of 647 K. pneumoniae isolates, $4.79 \%$ were found to be resistant against carbapenems and hence were KPC positive, which is 3 times higher than that found in 
Detection of Klebsiella pneumoniae Carbapenemase (KPC) and Metallo-Beta Lactamase (MBL) Producing Gram Negative Bacteria Isolated from Different Clinical Samples in A Transplant Center, Kathmandu, Nepal

India, where only $1.70 \% \mathrm{KPC}$ positive K. pneumoniae isolates were identified [54]. Another reported $E$ coli producing carbapenemase from the thirteen patients during their study in the hospitals. The KPC-encoding plasmids were highly related in 3 of them, suggesting the occurrence of their interspecies transfer [55].

Recently a study described a blaKPC -carrying plasmid that was common to two different species of Enterobacteriaceae (Citrobacter freundii and Klebsiella oxytoca) isolated in the same hospital. The bla ${ }_{\mathrm{KPC1} / 2}$ gene was located on $10 \mathrm{~kb}$ Tn3-type transposon (named $\mathrm{Tn}_{4401}$ ) and was identified on plasmids of different size. This transposon possesses two unrelated insertion sequences (IS) elements ISKpn6 and ISKpn 7. This phenomenon suggests that the gene can also move into different plasmids by insertion transposon [56]. Genetic structure (in detail) was described in Klebsiella pneumonia in France. According to 45 isolates of bla KPC were obtained from 21 cities in 11 countries across four continents [57].

A study conducted in central Nepal, a total of $18.98 \%(41 / 216)$ of $E$ coli and $21.08 \%$ (39/185) of K. pneumoniae were initially screened for carbapenemase production on the basis of their reduced susceptibility to meropenem or ertapenem by disc diffusion test. All the screening positive isolates of E. coli and K. pneumoniae were found to be positive for carbapenemase production by MHT. In the same study, E. coli $(\mathrm{n}=216)$ and K. pneumoniae $(\mathrm{n}=185)$ were recovered from various clinical samples in clinical microbiology laboratory of College of Medical Sciences, teaching hospital in central Nepal. Out of 216 E. coli isolates, a total of 41 isolates $(18.98 \%)$ and out of $185 \mathrm{~K}$. pneumoniae isolates, a total of 39 isolates $(21.08 \%)$ were initially screened for carbapenemase production on the basis of their reduced susceptibility to meropenem or ertapenem by disc diffusion test. All the screening positive isolates of $E$. coli and K. pneumoniae were found to be positive for carbapenemase production by MHT. However, 3 (7.31\%) isolates of $E$. coli and $4(10.26 \%)$ isolates of $K$. pneumoniae gave weakly positive reactions in MHT. This finding showed MBL production in a considerable number of E. coli and K. pneumoniae isolates with MDR and pandrug-resistant phenotypes [27].

\section{Conclusion}

The present study focused at the carbapenemase production among Gram- negative isolates in Human Organ Transplant Center Bhaktapur, Nepal using the phenotypic confirmatory test. Multidrug resistance to the antibiotics tested was also seen more among carbapenemase producer than the non-producers. E. coli and $K$. pneumoniae were found to be the predominant etiological agents isolated in the hospital. The antibiotic resistivity profile showed the significant incidence of the KPC and MBL in clinical settings. The third-generation cephalosporin was ineffective; thereby demand- ing new generation of novel antibiotics. The study shows the increasing trend of the multidrug resistant isolates with either of the resistance mechanisms. The threat of therapeutic failure has been increasing day by day. Thus, the early detection of $\beta$-lactamases producing isolates is important for the reduction of mortality rates and also to avoid circulation of such strains within the hospital.

\section{Acknowledgements}

We would like to express our sincere gratitude and admiration to all the staffs and faculties of Central Department of Microbiology, Tribhuvan University, Kirtipur and Human Organ Transplantation Centre, Bhaktapur for their support and guidance to complete this study.

\section{Funding}

This study has funded by departmental fund of Central Department of Microbiology, Tribhuvan University, Kirtipur, Kathmandu.

\section{Availability of data and materials}

All data pertaining to this study are within the manuscript.

\section{Author's contributions}

All the authors made substantial contribution to the study. KD collected samples, investigated and recorded the laboratory findings. KRR, BKA, SS and MRB advised and formulated the methodology for the study. BD and KRR drafted this manuscript. KRR and BD made subsequent version of revision and amend this manuscript. All authors read and approved the final version of manuscript.

\section{Competing Interests}

All the authors declared that they have no competing interests.

\section{Bibliography}

1. Bodro M., et al. "Impact of antibiotic resistance on the development of recurrent and relapsing symptomatic urinary tract infection in kidney recipients". American Journal of Transplantation 15.4 (2015): 1021-1027.

2. Origüen J., et al. "Progressive increase of resistance in Enterobacteriaceae urinary isolates from kidney transplant recipients over the past decade: narrowing of the therapeutic options". Transplant Infectious Disease 18.4 (2016): 575-584.

3. Kahan JS., et al. "Thienamycin, a new beta-lactam antibiotic. I. Discovery, taxonomy, isolation and physical properties". The Journal of Antibiotics 32 (1979): 1-12.

4. Chen HY and Livermore DM. "In-vitro activity of biapenem, compared with imipenem and meropenem, against Pseudomonas aeruginosa strains and mutants with known resistance mechanisms". Journal of Antimicrobial Chemotherapy 33 (1994): 949-958. 
5. Livermore DM., et al. "Activity of the anti-MRSA carbapenem razupenem (PTZ601) against Enterobacteriaceae with defined resistance mechanisms". Journal of Antimicrobial Chemotherapy 64 (2009): 3.

6. Budak S., et al. "Enterik gramnegatif bakterilerde laboratuvardan klinige karbapenemazlar". Mediterranean Journal of Infection Microbes and Antimicrobials 1 (2012): 1.

7. Russell SL., et al. "Early life antibiotic-driven changes in microbiota enhance susceptibility to allergic asthma". EMBO Reports 13 (2012): 440-447.

8. Bush K and Bradford PA. “ $\beta$-Lactams and $\beta$-Lactamase Inhibitors: An Overview". Cold Spring Harbor Perspectives in Medicine 6.8 (2016): a025247.

9. Queenan AM and Bush K. "Carbapenemases: the versatile $\beta$-lactamases". Clinical Microbiology Reviews 20 (2007): 440458.

10. Gupta N., et al. "Carbapenem-resistant Enterobacteriaceae: Epidemiology and prevention". Clinical Infectious Diseases 53 (2011): 60-67.

11. Nordmann P., et al. "Does broad-spectrum $\beta$-lactam resistance due to NDM-1 herald the end of the antibiotic era for treatment of infections caused by gram-negative bacteria?" Journal of Antimicrobial Chemotherapy 66 (2011): 689-692.

12. Paterson DL. "Resistance in gram-negative bacteria: Enterobacteriaceae". American Journal of Infection Control 34.5 (2006) 64-73.

13. Rajput R and Mishra AP. "A review on biological activity of quinazolinones". International Journal of Pharmaceutical Sciences 4.2 (2012): 66-70.

14. Yong D., et al. "Characterization of a new metallo beta lactamase gene, bla(NDM 1),and a novel erythromycin esterase gene carried on a unique genetic structure in Klebsiella pneumoniae sequence type 14 from India". Antimicrob Agents Chemother 53 (2009): 5046-5054.

15. Acar JF and Minozzi C. "Role of $\beta$-lactamases in the resistance of gram-negative bacilli to $\beta$-lactam antibiotics". Reviews of Infectious Diseases 8 (1986): 482-486.

16. Samanta I., et al. "Approaches to characterize extended spectrum beta-lactamase/beta-lactamase producing Escherichia coli infection". Genetics and Evolution 36 (2015): 224-230.

17. Kitchel B., et al. "Molecular epidemiology of KPC-producing Klebsiella pneumoniae isolates in the United States: clonal expansion of multilocus sequence type 258". Antimicrobial Agents and Chemotherapy 53 (2009): 3365-3370.
18. Page MI and Badarau A. "The Mechanisms of Catalysis by Metallo $\beta$-Lactamases". Bioinorganic Chemistry and Applications (2008): 576297.

19. Patel G., et al. "Outcomes of carbapenem-resistant Klebsiella pneumoniae infection and the impact of antimicrobial and adjunctive therapies". Infection Control and Hospital Epidemiologylogy 29 (2008): 1099-1106.

20. Papadimitriou-Olivgeris M., et al. "Risk factors for kpc-producing Klebsiella pneumoniae enteric colonization upon ICU admission". Journal of Antimicrobial Chemotherapy 67 (2012): 2976-2981.

21. Tumbarello M., et al. "Predictive models for identification of hospitalized patients harboring kpc-producing Klebsiella pneumoniae". Antimicrob Agents Chemother (Bethesda) 58 (2014): 3514-3520.

22. Mishra SK., et al. "Metallo-beta-lactamase producing Gramnegative bacterial isolates". Journal of Nepal Health Research Council 10 (2012): 208-213.

23. Shrestha S., et al. "Prevalence of nosocomial lower respiratory tract infections caused by Multi-drug resistance pathologens". Journal of the Institute of Medicine 6 (2012): 28-32.

24. Khanal S., et al. " $\beta$-Lactamase-Producing Multidrug-Resistant Bacterial Pathogens from Tracheal Aspirates of Intensive Care Unit Patients at National Institute of Neurological and Allied Sciences, Nepal". International Scholarly Research Notices Microbiology (2013): 847569.

25. Tada T., et al. "NDM-1 Metallo- $\beta$-Lactamase and ArmA $16 \mathrm{~S}$ rRNA methylase producing Providencia rettgeri clinical isolates in Nepal". BMC Infectious Diseases 14 (2014): 56.

26. Ansari S., et al. "Community acquired multi-drug resistant clinical isolates of Escherichia coli in a tertiary care center of Nepal". Antimicrobial Resistance and Infection Control 4 (2015):15.

27. Bora A., et al. "Incidence of metallo-beta lactamase producing clinical isolates of Escherichia coli and Klebsiella pneumoniae in central Nepal". BMC Research Notes 7 (2014): 557.

28. Karn S., et al. "Prevalence of carbapenem resistant bacterial strains isolated from different clinical samples: study from a tertiary care hospital in Kathmandu, Nepal". Journal of Biomedical Science 3 (2016): 11-15.

29. Van Duin D., et al. "Multidrug-resistant gram-negative bacteria infections in solid organ transplantation". American Journal of Transplantation 13 (2013): 31-41.

30. Cheesbrough M. "District laboratory practice in tropical countries". Cambridge University Press (2006). 
31. Forbes BA., et al. "Bailey and Scott's. Diagnostic microbiology".12th edition, Mosby Elsevier (2007).

32. Ramadan DS., et al. "Detection of ESBL producing bacteria in cases of UTI in pediatric department of Benha university hospital". Egyptian Journal of Medical Microbiology 25 (2016): 77-84.

33. Magiorakos AP., et al. "Multidrug-resistant, extensively drugresistant and pandrug-resistant bacteria: an international expert proposal for interim standard definitions for acquired resistance". Clinical Microbiology and Infection 18 (2012): 268-281.

34. Giske CG., et al. "A sensitive and specific phenotypic assay for detection of metallo- $\beta$-lactamases and KPC in Klebsiella pneumoniae with the use of meropenem disks supplemented with amino-phenylboronic acid, dipicolinic acid and cloxacillin". Clinical Microbiology and Infection 17 (2011): 552-556.

35. Tsakris A., et al. "Evaluation of boronic acid disk tests for differentiating KPC-possessing Klebsiella pneumoniae isolates in the clinical laboratory". Journal of Clinical Microbiology 47 (2009): 362-367.

36. Pasteran F., et al. "Sensitive screening tests for suspected class A carbapenemase production in species of Enterobacteriaceae". Journal of Clinical Microbiology 47 (2009): 16311639.

37. Gautam K., et al. "Phenotypic Detection of Carbapenemases and $\beta$-lactamases induced Carbapenem Resistance in Enterobacteriaceae". The Pharmaceutical and Chemical Journal 2 (2015): 1-7.

38. Pathak J and Pokharel N. "Multidrug resistant and extended spectrum $\beta$-lactamase (ESBL) isolates from different clinical specimens". International Journal of Scientific and Research 5 (2015):1-5

39. Poudyal S., et al. "Extended Spectrum Beta-lactamase producing multidrug resistant clinical bacterial isolates at National Public Health Laboratory, Nepal". Nepal Medical College Journal 13 (2011): 34-38.

40. Hamid ME., et al. "Prevalence of bacterial pathogens in Aseer Region, Kingdom of Saudi Arabia: Emphasis on antimicrobial susceptibility of Staphylococcus aureus". Oman Medical Journal 26 (2011): 368-370.

41. Bhattarai R. "Detection of Metallo-beta-lactamase producing Pseudomonas aeruginosa in clinical specimens of patients visiting B and B hospital. M.SC Dissertation submitted to the GoldenGate International College". Department of Microbiology, Kathmandu, Nepal (2014).
42. Raut S., et al. "Prevalence of Extended Spectrum Beta-Lactamases among Escherichia coli and Klebsiella spp isolates in Manipal Teaching Hospital, Pokhara, Nepal". Journal of Microbiology and Infectious Diseases 5.2 (2015): 69-75.

43. Abbott SL., et al. "Klebsiella, Enterobacter, Citrobacter, Serratia, Plesiomonas, and other Enterobacteriaceae". In Manual of clinlical microbiology. Washington DC. ASM Press (2007): 698-715.

44. Farmer J., et al. "Biochemical identification of new species and biogroups of Enterobacteriaceae isolated from clinical specimens". Journal of Clinical Microbiology 21 (1985): 46-76.

45. Sharma M., et al. "Prevalence and antibiogram of Extended Spectrum $\beta$-Lactamase (ESBL) producing Gram negative bacilli and further molecular characterization of ESBL producing Escherichia coli and Klebsiella spp". Journal of Clinical and Diagnostic Research 7.10 (2013): 2173-2177.

46. Baral P. "Multidrug resistance among various clinical bacterial isolates and production of different types of $\beta$-lactamases with subsequent transfer mechanism by plasmid DNA analysis". M.Sc. Dissertation submitted to the Central Department of Microbiology, Tribhuvan University, Kathmandu, Nepal (2008).

47. Livermore DM. " $\beta$-Lactamases in Laboratory and Clinical Resistance". Clinical Microbiology Reviews 8 (1995): 557-558.

48. Souli M., et al. "Emergence of extensively drug-resistant and pandrug-resistant Gram-negative bacilli in Europe". Eurosurveillance 13.47 (2008): 19045.

49. Shenoy KA., et al. "Phenotypic identification and molecular detection of blaNDM-1 gene in multidrug resistant Gramnegative bacilli in a tertiary care centre". The Indian Journal of Medical Research 139 (2014): 625-631.

50. Nordmann P., et al. "Does broad-spectrum $\beta$-lactam resistance due to NDM-1 herald the end of the antibiotic era for treatment of infections caused by gram-negative bacteria?" Journal of Antimicrobial Chemotherapy 66.4 (2011): 689-692.

51. Poirel L., et al. "Carbapenem-hydrolyzing metallo- $\beta$-lactamase from a nosocomial isolate of Pseudomonas aeruginosa in France". Emerging Infectious Disease Journal 6 (2000): 84-85.

52. Datta S., et al. "A ten-year analysis of multi-drug resistant blood stream infections caused by Escherichia coli and Klebsiella pneumoniae in a tertiary care hospital". Indian Journal of Medical Research 135.6 (2012): 907-912.

53. Wattal C., et al. "Surveillance of multidrug resistant organisms in tertiary care hospital in Delhi, India". Journal of the Association of Physicians of India 58 (2010): 32-36. 
54. Kumarasamy KK., et al. "Emergence of a new antibiotic resistance mechanism in India, Pakistan, and the UK: a molecular, biological, and epidemiological study". The Lancet Infectious Diseases 10 (2010): 597-602.

55. Kim YA., et al. "Features of Infections Due to Klebsiella pneumoniae Carbapenemase-Producing Escherichia coli: Emergence of Sequence Type 131". Clinical Infectious Diseases 55 (2012): 224-231.

56. Rasheed JK., et al. "New Delhi Metallo- $\beta$-Lactamase-producing Enterobacteriaceae United States". Emerging Infectious Diseases 19.6 (2013): 870-878.

57. Stoesser N., et al. "Genomic epidemiology of global Klebsiella pneumoniae carbapenemase (KPC) producing Escherichia coli". Scientific Reports 7.1 (2017): 5917.

Volume 2 Issue 12 December 2019

(C) All rights are reserved by Komal Raj Rijal., et al. 